\title{
Um Estudo sobre a Influência da Sistematização da Construção de Modelos de Casos de Uso na Contagem dos Pontos de Casos de Uso
}

\author{
Anderson Belgamo ${ }^{1}$ e Sandra Fabbri \\ Departamento de Computação \\ Universidade Federal de São Carlos \\ Rodovia Washington Luis, $\mathrm{km} 235$ \\ Cx. Postal 676, 13565-905 \\ São Carlos - SP - Brasil \\ \{belgamo, sfabbri\}@dc.ufscar.br
}

\begin{abstract}
Resumo
Este artigo apresenta um estudo sobre a influência exercida na contagem dos Pontos de Casos de Uso, quando esta é realizada com base em modelos construídos com uma maior sistemática e padronização. Essa sistemática na construção de Modelos de Casos de Uso é obtida com a aplicação das técnicas de leitura GUCCRA Guidelines for Use Case Construction and Requirements document Analysis. Esse estudo foi realizado com base em Modelos de Casos de Uso elaborados durante um experimento cujo objetivo foi avaliar essas técnicas de leitura em relação a uma abordagem Ad-Hoc de construção. As análises realizadas com os Pontos de Casos de Uso computados para os modelos gerados com as técnicas de leitura mostram que esses valores são mais próximos do valor correspondente a um Modelo Oráculo do que aqueles decorrentes dos modelos gerados pela abordagem Ad-Hoc. Assim, a elaboração de casos de uso mais padronizados e menos dependentes da subjetividade do projetista, como mostraram os resultados do experimento, podem melhorar a qualidade dos Pontos de Casos de Uso computados para os modelos.
\end{abstract}

Palavras-Chave: pontos de casos de uso, construção de modelos de casos de uso, técnicas de leitura.

\begin{abstract}
This paper presents a study on the influence in Use Case Points derived from models constructed with a more systematic and standardized process. This systematization on Use Case Model construction is reached by applying the reading techniques named GUCCRA - Guidelines for Use Case Construction and Requirements document Analysis. This study was realized based on use case models constructed during an experiment aiming at evaluating the reading techniques in relation to the Ad-Hoc approach. The analysis carried out with the Use Cases Points computed for the models constructed with the reading techniques showed that these values are nearest to the correspondent value of the Oracle Model than the ones derived from the Ad-Hoc approach. Therefore, the elaboration of use cases that are more standardized and less dependent of the designer subjectivity, as it was showed by the experiment results, can improve the quality of the Use Case Points associated with those models.
\end{abstract}

Keywords: use case points, use case models construction, reading techniques.

\section{Introdução}

Uma das características imprescindíveis de qualidade de um software é que ele esteja em conformidade com os requisitos do cliente. Entende-se por cliente, os vários tipos de clientes que um software pode ter desde sua concepção, ou seja, o usuário do software, o

\footnotetext{
${ }^{1}$ Bolsista da CAPES
} 
desenvolvedor e a própria organização. Embora todos esses clientes olhem o software sob uma ótica diferente, um requisito comum a eles é o planejamento, o qual é também um item fundamental em relação aos modelos de qualidade de processo, como por exemplo, o CMM [1], CMMI [2] e Norma ISO 15504 [3]. Para alguns desses modelos, a primeira iniciativa de melhoria do processo de desenvolvimento de software se dá justamente nos aspectos administrativos, entre os quais está o Planejamento, como uma área chave para que a empresa saia de um nível totalmente desorganizado e caótico.

A atividade de Planejamento é, essencialmente, baseada em dados históricos que, quanto mais acurados, mais podem aumentar a precisão das estimativas baseadas neles. Para realizar a atividade de Planejamento, várias técnicas (métricas) podem ser utilizadas para medir o tamanho ou a complexidade do software. Essas medidas podem, posteriormente, servir como entrada para modelos de estimativas que irão produzir dados sobre o custo, prazo e esforço de desenvolvimento. Dentre essas técnicas, podem-se citar a Análise de Pontos por Função (APF), criada por Alan Albrecht [4], que é utilizada para calcular a complexidade do software e, indiretamente, o seu tamanho, e o modelo COnstructive COst MOdel (COCOMO) [5], que é utilizado para fazer as estimativas e que foi evoluído para um modelo mais abrangente, chamado COCOMO II. Este último pode receber como entrada as seguintes informações de tamanho: Pontos por Objetos (PO), Pontos por Função (PF) e linhas de código fonte (LOC) [6]. Outra técnica que pode ser utilizada para gerar estimativas, e que é explorada no contexto deste artigo, é a de Pontos de Casos de Uso (PCU) [7], os quais podem ser convertidos para Pontos por Função, de acordo com Andrade [8].

Os Pontos de Casos de Uso são gerados com base em um Modelo de Casos de Uso, o qual, por sua vez, é gerado a partir do Documento de Requisitos. Assim, para que os Pontos de Casos de Uso sejam o mais acurado possível, como é necessário para um conjunto de dados históricos, é essencial que eles sejam computados a partir de um modelo que retrate, da forma mais apropriada e fiel possível, os requisitos do cliente.

Assim, neste artigo comentam-se as técnicas de leitura GUCCRA - Guidelines for Use Case Construction and Requirements document Analysis [9] que também visam, do ponto de vista de construção, o apoio à elaboração de Modelos de Casos de Uso e, do ponto de vista de análise, a deteç̧ão de defeitos em documentos de requisitos. Assim, essas técnicas fornecem diretrizes para a construção de Modelos de Casos de Uso mais padronizados, sendo que, simultaneamente à elaboração dos mesmos, o documento de requisitos que está sendo utilizado seja inspecionado.

Conseqüentemente, podendo-se, com o apoio das técnicas GUCCRA, gerar casos de uso mais padronizados e menos dependentes da subjetividade e experiência do projetista, a qualidade dos PCU e, portanto, do planejamento realizado para o desenvolvimento do software pode aumentar.

Com base nesse contexto, o objetivo deste artigo é apresentar uma análise dos Pontos de Casos de Uso derivados de Modelos de Casos de Uso que foram produzidos em um experimento que teve por objetivo verificar a viabilidade de aplicação das técnicas GUCCRA, além de comparar os modelos gerados com sua aplicação, com modelos gerados com a aplicação de uma abordagem Ad-hoc. Com essa análise, dão-se evidências da influência da qualidade dos Modelos de Casos de Uso na qualidade dos Pontos de Casos de Uso e, conseqüentemente, no planejamento do software.

Este artigo está organizado da seguinte forma: na Seção 2 apresentam-se as técnicas GUCCRA, na Seção 3 a síntese do experimento executado para avaliar as técnicas GUCCRA e do qual foram extraídos os Modelos de Casos de Uso usados neste artigo para avaliar os Pontos de Casos de Uso, na Seção 4 a técnica de Pontos de Casos de Uso, na Seção 5 a 
contribuição das técnicas GUCCRA na contagem de Pontos de Casos de Uso e, na Seção 6, as conclusões.

\section{As Técnicas de Leitura GUCCRA}

A criação de um Modelo de Casos de Uso não é algo trivial e de fácil padronização por todos os envolvidos na modelagem de requisitos. Uma das principais dificuldades vem do fato de decidir quais são os casos de uso do sistema e como representar as especificações dos casos de uso identificados. A literatura existente sobre modelagem de requisitos com casos de uso não fornece diretrizes para determinação dos casos de uso de forma que modelos semelhantes possam ser gerados, independentemente da pessoa que esteja desenvolvendo o modelo. Com o objetivo de contribuir nessa perspectiva, as técnicas de leitura GUCCRA fornecem diretrizes para a elaboração de Modelos de Casos de Uso sendo que, simultaneamente à sua aplicação, o documento de requisitos (que é entrada para a aplicação das mesmas) também é avaliado com o objetivo de identificação de defeitos. Este aspecto não será tratado no âmbito do artigo, já que no contexto do mesmo o que interessa é a sistematização dos Modelos de Casos de Uso.

As duas leituras que compõem as técnicas GUCCRA são: Actor-Goal Reading Technique (AGRT) e Use Case Reading Technique (UCRT), as quais são comentadas nas próximas seções.

\subsection{Técnica de Leitura para identificação de Atores e Objetivos}

$\mathrm{O}$ artefato de entrada utilizado pela técnica AGRT (Actor-Goal Reading Technique) é o documento de requisitos e ela tem por objetivo identificar os candidatos a atores e seus respectivos objetivos de utilização do sistema, fazendo isso através da marcação dos substantivos e verbos, respectivamente, no documento de requisitos. O artefato de saída gerado pela aplicação da técnica AGRT é o Formulário Ator x Objetivo (FAO), cuja estrutura pode ser vista na Figura 1.

\begin{tabular}{|l|l|l|}
\multicolumn{1}{c|}{ Ator } & \multicolumn{1}{|c|}{ Objetivo } & \multicolumn{1}{|c|}{ Referência } \\
\hline $\begin{array}{l}\text { Atores } \\
\text { identificados }\end{array}$ & $\begin{array}{l}\text { Objetivos identificados para } \\
\text { cada ator }\end{array}$ & $\begin{array}{l}\text { O número da seção ou número do requisito que } \\
\text { contém os objetivos dos atores }\end{array}$ \\
\hline
\end{tabular}

Figura 1. Formulário Ator X Objetivo (FAO)

O Formulário Ator x Objetivo foi baseado na Lista Ator x Objetivo [10] que, segundo Cockburn tem o propósito de ser um ponto inicial para discussão com os stakeholders quando a elicitação de requisitos está sendo realizada. Ao final da aplicação dessa leitura, esse formulário possui todos os atores e respectivos objetivos identificados no documento de requisitos.

Durante a identificação dos atores e de seus objetivos para preenchimento do FAO a técnica instrui o projetista a relatar defeitos no documento de requisitos, os quais são identificados pelo fato de não permitirem ou não darem o suporte necessário para que o FAO possa ser preenchido. 


\subsection{Técnica de Leitura para identificação de Casos de Uso}

A técnica UCRT (Use Case Reading Technique) tem por objetivo criar o Modelo de Casos de Uso propriamente dito. Com base no FAO, a técnica UCRT tem como propósito entender os objetivos de cada ator identificado nesse formulário, com o intuito de criar casos de usos preliminares e especificá-los. Nessa etapa, são identificados possíveis relacionamentos entre os casos de uso de forma que eles possam ser agrupados gerando um único caso de uso apenas ou serem fatorados gerando dois ou mais casos de uso.

Os casos de uso criados são baseados na UML [11] e utilizam associações com os estereótipos $<<$ include $>>$ e/ou $<<$ extend $>>$. O template de especificação dos casos de uso adotado foi baseado em templates encontrados na literatura $[7,10,12,13]$, conforme mostra a Figura 2.

Cada passo criado durante a especificação dos cursos normal e alternativo dos casos de uso deve descrever uma das seguintes situações [10]:

- Uma interação (exemplo: Cliente entra com endereço).

- Um passo de validação para proteger um interesse de um stakeholder (exemplo: Sistema valida código)

- Uma mudança interna para satisfazer um interesse de um stakeholder (exemplo: Sistema deduz total da conta)

Para descrição das situações mostradas acima, algumas sugestões de escritas de passos $[10,14]$ devem ser seguidas e são mostradas na Tabela 1.

\begin{tabular}{|c|c|c|c|}
\hline & Especificação do Caso de Uso & Número: & Número do Caso de Uso \\
\hline Nome do Caso de Uso & \multicolumn{3}{|l|}{ Nome do caso de uso criado } \\
\hline Descrição ou Resumo & \multicolumn{3}{|l|}{ Pequena descrição do caso de uso } \\
\hline Ator Participante & \multicolumn{3}{|c|}{ Atores primários relacionados ao caso de uso } \\
\hline Ator Operador & \multicolumn{3}{|c|}{ Atores secundários associados ao caso de uso } \\
\hline Ator Genérico & \multicolumn{3}{|c|}{ Ator genérico criado pela análise dos atores participantes. } \\
\hline Pré-Condição & \multicolumn{3}{|c|}{$\begin{array}{l}\text { Condições que devem ser verdadeiras para que o caso de uso possa ser } \\
\text { realizado }\end{array}$} \\
\hline Curso Normal & \multicolumn{3}{|c|}{ Corresponde a um fluxo de eventos } \\
\hline Curso Alternativo & \multicolumn{3}{|c|}{$\begin{array}{l}\text { Corresponde a fluxos de eventos, porém mostrando os caminhos menos } \\
\text { comuns de acontecer }\end{array}$} \\
\hline Evento Disparador & \multicolumn{3}{|c|}{ Descreve o critério de entrada para o caso de uso sendo especificado } \\
\hline Include & \multicolumn{3}{|c|}{ Número dos casos de uso relacionados pelo estereótipo $<<$ include $>>$} \\
\hline Extend & \multicolumn{3}{|c|}{ Número dos casos de uso relacionados pelo estereótipo $<<$ extend $>>$} \\
\hline Requisitos Funcionais & \multicolumn{3}{|c|}{ Os números dos requisitos funcionais associados ao caso de uso criado } \\
\hline Requisitos Não-Funcionais & \multicolumn{3}{|c|}{ Os números dos requisitos não funcionais associados ao caso de uso criado } \\
\hline Autor & \multicolumn{3}{|l|}{ A pessoa que criou a especificação } \\
\hline Data & \multicolumn{3}{|l|}{ A data da criação da especificação } \\
\hline Versão & \multicolumn{3}{|c|}{ Código associado à versão da especificação } \\
\hline
\end{tabular}

Figura 2. Template de especificação de casos de uso

Os defeitos encontrados durante a aplicação da UCRT referem-se, principalmente, à falta de informação suficiente, no documento de requisitos, para que os casos de uso sejam devidamente especificados.

Ao final da aplicação da técnica UCRT é obtido um Modelo de Casos de Uso que retrata os requisitos dos clientes expressos no documento de requisitos. 
Tabela 1. Sugestões de escrita de passos dos casos de uso criados

\begin{tabular}{|c|c|}
\hline Sugestão & Exemplo \\
\hline Use uma gramática simples & $\begin{array}{l}\text { Sujeito....verbo.....objeto direto.....frase preposicional } \\
\text { O sistema......deduz....... a quantidade.......do saldo da conta }\end{array}$ \\
\hline $\begin{array}{l}\text { Não especifique situações em } \\
\text { muito baixo nível. }\end{array}$ & $\begin{array}{l}\text { Não recomendado - baixo nível } \\
\text { - Cliente pressiona a tecla tab } \\
\text { - Cliente informa o número do CPF. } \\
\text { Recomendado } \\
\text { - Cliente entra com o número do CPF }\end{array}$ \\
\hline $\begin{array}{l}\text { Mostre a intenção do ator, não os } \\
\text { movimentos }\end{array}$ & $\begin{array}{l}\text { 1. Sistema requisita o nome } \\
\text { 2. Cliente informa o nome } \\
\text { 3. Sistema requisita CPF } \\
\text { 4. Cliente informa o } \mathrm{CPF} \\
\text { 5. Cliente clica no botão OK } \\
\text { 6. etc........ } \\
\text { Corrigindo: } 1 . \text { Cliente informa o nome e o CPF } \\
\text { 2. etc }\end{array}$ \\
\hline
\end{tabular}

\section{Síntese do Experimento para avaliação das técnicas de leitura GUCCRA}

O objetivo desta seção é mostrar que a aplicação das técnicas GUCCRA sistematiza a construção de Modelos de Casos de Uso. Embora os objetivos das técnicas GUCCRA sejam a construção de Modelos de Casos de Uso e a identificação de defeitos em documentos de requisitos, os resultados do experimento aqui apresentado estão baseados somente na compilação dos dados referentes à construção dos Modelos de Casos de Uso.

O principal objetivo do experimento foi verificar a contribuição das técnicas GUCCRA no sentido de analisar quão próximos seriam os Modelos de Casos de Uso elaborados pelos participantes, utilizando essas técnicas e utilizando a abordagem Ad Hoc, ou seja, sem um procedimento definido.

O experimento foi realizado com 18 estudantes, divididos em seis grupos de três, da disciplina de Engenharia de Software dos cursos de Bacharelado em Ciência da Computação e Engenharia da Computação da Universidade Federal de São Carlos. Nenhum participante do experimento possuía conhecimento prévio de modelagem de requisitos através de casos de uso. O projeto experimental foi aplicado do seguinte modo (Figura 3).

\begin{tabular}{|c|c|c|c|c|c|c|c|}
\hline & $\begin{array}{c}\text { Grupo A } \\
\mathbf{3} \\
\text { participantes }\end{array}$ & $\begin{array}{c}\text { Grupo B } \\
\mathbf{3} \\
\text { participantes }\end{array}$ & $\begin{array}{c}\text { Grupo C } \\
\mathbf{3} \\
\text { participantes }\end{array}$ & $\begin{array}{c}\text { Grupo D } \\
\mathbf{3} \\
\text { participantes }\end{array}$ & $\begin{array}{c}\text { Grupo E } \\
\mathbf{3} \\
\text { participantes }\end{array}$ & $\begin{array}{c}\text { Grupo F } \\
\mathbf{3} \\
\text { participantes }\end{array}$ & \\
\hline \multirow[t]{2}{*}{ Ad-Hoc } & \multicolumn{6}{|c|}{ Treinamento em Modelagem de Casos de Uso (Ad-Hoc) } & \multirow{2}{*}{$\begin{array}{c}\text { Primeira } \\
\text { Sessão }\end{array}$} \\
\hline & Doc. Req. D & Doc. Req. F & Doc. Req. B & Doc. Req. A & Doc. Req. C & Doc. Req. E & \\
\hline \multirow[t]{2}{*}{ AGRT } & \multicolumn{6}{|c|}{ Treinamento em Actor Goal Reading Technique } & \multirow{2}{*}{$\begin{array}{c}\text { Segunda } \\
\text { Sessão }\end{array}$} \\
\hline & Doc. Req. C & Doc. Req. D & Doc. Req. F & Doc. Req. E & Doc. Req. A & Doc. Req. B & \\
\hline \multirow[t]{2}{*}{ UCRT } & \multicolumn{6}{|c|}{ Treinamento em Use Case Reading Technique } & \multirow{2}{*}{$\begin{array}{c}\text { Terceira } \\
\text { Sessão }\end{array}$} \\
\hline & Doc. Req. C & Doc. Req. D & Doc. Req. F & Doc. Req. E & Doc. Req. A & Doc. Req. B & \\
\hline
\end{tabular}

Figura 3. Projeto Experimental 
Os seis documentos de requisitos utilizados no experimento foram criados pelos grupos e foram baseados no padrão IEEE [15] de documentação de requisitos, no qual algumas seções importantes foram consideradas, como por exemplo, Propósito, Escopo, Definições, Perspectiva do Produto, Funções do Produto, Características dos Usuários, Suposições e Dependências, Requisitos Funcionais e Requisitos Não-Funcionais.

É importante observar, pelo projeto experimental, que o grupo que utilizou um documento de requisitos para aplicação da abordagem Ad-Hoc utilizou um outro documento para aplicação das técnicas GUCCRA. Essa decisão teve o objetivo de fazer com que nenhum grupo utilizasse o documento de requisitos criado por ele mesmo e também evitou que um prévio conhecimento do documento de requisitos interferisse na aplicação das técnicas.

Para efeito de análise dos resultados, os casos de uso criados pelas técnicas GUCCRA e Ad-Hoc foram comparados aos Modelos Oráculo de cada documento de requisitos, os quais foram construídos com o apoio das técnicas GUCCRA por uma pessoa que tem domínio das mesmas.

Os resultados do experimento mostraram que o tempo de aplicação das técnicas GUCCRA é maior que o tempo de aplicação da abordagem Ad-Hoc para todos os documentos de requisitos utilizados. No entanto, a efetividade na identificação dos casos de uso, em relação ao Modelo Oráculo, também foi maior em todos os casos.

Com o objetivo de verificar se participantes que utilizaram o mesmo documento de requisitos tiveram um desempenho diferente com as técnicas GUCCRA e abordagem Ad-Hoc analisou-se a efetividade e eficiência dos participantes levando em consideração a interação da técnica com o documento de requisitos, somente da técnica e somente do documento de requisitos. Para tal, utilizou-se a estatística ANOVA e os resultados obtidos são comentados a seguir:

- A interação da técnica com o documento de requisitos influenciou os resultados obtidos tanto no que se refere à efetividade quanto à eficiência.

- O efeito da técnica influenciou os resultados obtidos no que se refere à efetividade, mas no que se refere à eficiência não foi possível concluir se a técnica influenciou ou não.

- O efeito do documento de requisitos influenciou os resultados obtidos no que se refere à eficiência, mas no que se refere à efetividade não foi possível concluir se o documento influenciou ou não.

Além da utilização da estatística ANOVA, outras análises foram feitas observando-se a quantidade de casos de uso coincidentes entre os modelos elaborados pelos participantes e os respectivos Modelos Oráculo, a quantidade de casos de uso dos Modelos Oráculo que não foram encontrados nos modelos dos participantes e o número de casos de uso identificados nos modelos construídos pelos participantes e que não constaram nos Modelos Oráculo.

Resumindo, os resultados experimentais mostraram evidências de que as técnicas GUCCRA, quando analisadas sob o ponto de vista de construção de Modelos de Casos de Uso, padronizam a identificação dos casos de uso, pois os casos de uso identificados são semelhantes àqueles dos Modelos Oráculo; melhoram a efetividade na identificação de casos de uso, pois o número de casos de uso identificados pelas técnicas e não pertencentes aos Modelos Oráculo é menor com a aplicação das técnicas GUCCRA em relação à abordagem Ad-Hoc, e auxiliam na tomada de decisão em relação aos aspectos de agrupamento ou separação da funcionalidade referente aos casos de uso, identificando relacionamentos entre os casos de uso com estereótipos $<<$ include $>>$ e/ou $<<$ extend $>>$. 


\section{Pontos de Casos de Uso}

Os Pontos de Casos de Uso (PCU) foram criados em 1993 por Gustav Karner da empresa Objectory $A B$ (adquirida mais tarde pela Rational Software Corporation) e têm como objetivo estimar projetos de software baseados em casos de uso [7].

A filosofia dos Pontos de Casos de Uso é baseada na definição da Análise de Pontos por Função (APF), na qual a funcionalidade vista pelo usuário é a base para a estimativa do tamanho do software. Assim como a Análise de Pontos por Função, essa métrica também permite a contagem dos Pontos de Casos de Uso não ajustados e ajustados.

A contagem dos Pontos de Casos de Uso não ajustados é realizada em dois passos: classificação de atores e classificação de casos de uso.

Inicialmente, os atores identificados para o sistema são analisados com o intuito de classificá-los em um dos três tipos de complexidade, à qual é atribuído um fator de peso, conforme mostra a Tabela 2.

Tabela 2. Classificação de Atores

\begin{tabular}{|l|l|c|}
\hline \multicolumn{1}{|c|}{ Tipo do Ator } & \multicolumn{1}{c|}{ Descrição } & Fator de Peso \\
\hline Simples & Interface de Programa & 1 \\
\hline Médio & Interface interativa ou dirigia a protocolo & 2 \\
\hline Complexo & Interface gráfica & 3 \\
\hline
\end{tabular}

Um ator simples representa outro sistema com uma Interface de Aplicação (API) definida. Um ator médio representa um outro sistema interagindo através de um protocolo de comunicação, por exemplo TCP/IP. Um ator complexo é uma pessoa interagindo através de uma interface gráfica (GUI) ou uma página na internet.

Em seguida, os casos de usos identificados para o sistema são classificados em um dos três tipos de complexidade. Da mesma forma, para cada tipo de complexidade é atribuído um fator de peso, conforme mostra a Tabela 3. A base para decisão do tipo do caso de uso é o número de transações em um caso de uso, incluindo cursos alternativos. Para esse propósito, uma transação é definida como sendo um conjunto de atividades atômicas, as quais podem ser realizadas inteiramente ou não. Contar o número de transações pode ser feito contando-se os passos contidos nos cursos normal e alternativos das especificações dos casos de uso [16].

Tabela 3. Classificação de Casos de Uso

\begin{tabular}{|l|l|c|}
\hline \multicolumn{1}{|c|}{ Tipo do Caso de Uso } & \multicolumn{1}{|c|}{ Descrição } & Fator de Peso \\
\hline Simples & 3 ou menos transações & 5 \\
\hline Médio & 4 a 7 transações & 10 \\
\hline Complexo & Mais que 7 transações & 15 \\
\hline
\end{tabular}

Tanto para os atores como para os casos de uso o fator de peso deve ser multiplicado pela quantidade identificada de cada um para, posteriormente, ser realizada a somatória dos produtos obtidos, totalizando os valores não ajustados relativos aos atores e aos casos de uso. Para finalizar a contagem dos Pontos de Casos de Uso não ajustados é necessário realizar a somatória desses dois valores.

Da mesma forma como é feito na APF, em que o ajuste dos Pontos por Função é feito por meio de fatores de peso técnico, os Pontos de Casos de Uso, obtidos pela contagem dos atores e casos de uso, podem ser ajustados utilizando-se fatores de pesos técnicos e ambientais, como por exemplo, a complexidade do projeto e o nível de experiência dos participantes do projeto. Para cada fator (técnico e ambiental) um conjunto de sub-fatores é analisado com o 
intuito de atribuir um peso. O somatório dos pesos dos sub-fatores são utilizados para a obtenção dos Pontos de Casos de Uso ajustados.

Um ponto discutido em relação à técnica de Pontos de Casos de Uso proposta por Karner, diz respeito à não contagem de casos de uso com estereótipos $<<$ extend $>>\mathrm{e}<<$ include $>>$. Porém, essa característica foi avaliada na indústria e mostrou que a contagem desses tipos de casos de uso fornece uma estimativa mais próxima comparada ao esforço real gasto no desenvolvimento do software [16, 17]. Assim, é aconselhável que todos os casos de uso identificados sejam contados, de modo a evitar que os valores dos Pontos de Casos de Uso fiquem subestimados. Outra característica identificada nos estudos realizados por Anda [18] mostrou que a utilização de Pontos de Casos de Uso tende a reduzir a necessidade do conhecimento do especialista no processo de estimativa.

Ressalta-se que, de acordo com dados obtidos da aplicação dessa técnica em projetos acadêmicos e da indústria, Andrade [8] observou a necessidade de padrões pré-definidos, para que os desenvolvedores tenham um claro entendimento de como definir os casos de uso durante o processo de desenvolvimento de software. Segundo a autora, isso pode facilitar o processo de contagem dos Pontos de Casos de Uso.

\section{A melhoria na contagem dos PCU com modelos gerados com as Técnicas de Leitura GUCCRA}

Com o objetivo de avaliar se a qualidade dos PCU é influenciada por Modelos de Casos de Uso construídos pelas técnicas GUCCRA e abordagem Ad-Hoc, apresentam-se nesta seção as seguintes análises:

1) Uma comparação dos PCU computados para os modelos gerados com as técnicas GUCCRA e com a abordagem Ad-Hoc.

2) Uma avaliação dos PCU computados para os modelos gerados com as técnicas GUCCRA e abordagem Ad-Hoc em relação aos PCU computados para os Modelos Oráculo.

3) Uma avaliação dos PCU computados para os modelos gerados com as técnicas GUCCRA e abordagem Ad-Hoc em relação aos PCU computados para os Modelos Oráculo, mas considerando apenas os casos de uso dos modelos que coincidem com os casos de uso dos Modelos Oráculo.

Vale salientar que os PCU analisados foram os não-ajustados, uma vez que o valor de ajuste seria o mesmo, para cada documento de requisitos considerado, independentemente da técnica utilizada, pois esse ajuste leva em consideração fatores técnicos e ambientais, que são invariáveis para o mesmo sistema.

Nas próximas seções discutem-se os dados obtidos de acordo com os três pontos de análise estabelecidos anteriormente.

\subsection{Comparação dos PCU: GUCCRA X Ad-Hoc}

A análise apresentada nesta seção utilizou estatística descritiva, ou seja, é baseada em dados relativos à média, mediana, moda e desvio padrão relacionados aos PCU computados.

O objetivo dessa análise foi avaliar se os PCU obtidos para os modelos que utilizaram a mesma técnica, sejam as técnicas GUCCRA ou abordagem Ad-Hoc, eram próximos tanto no caso da própria técnica, como entre as técnicas.

Cada uma das Figuras de 4 a 9 está relacionada a um documento de requisitos utilizado no experimento. Elas apresentam um gráfico que mostra, para cada uma das técnicas utilizadas (GUCCRA e Ad-Hoc) a média, a mediana e a moda dos PCU dos modelos 
construídos com a referida técnica (vide legenda), o valor dos PCU dos Modelos Oráculos (linha tracejada) e o valor do desvio-padrão (linha contínua).

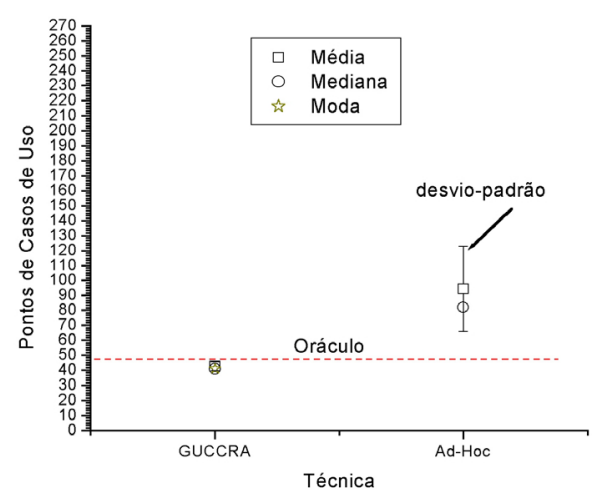

Figura 4. Documento de Requisitos A

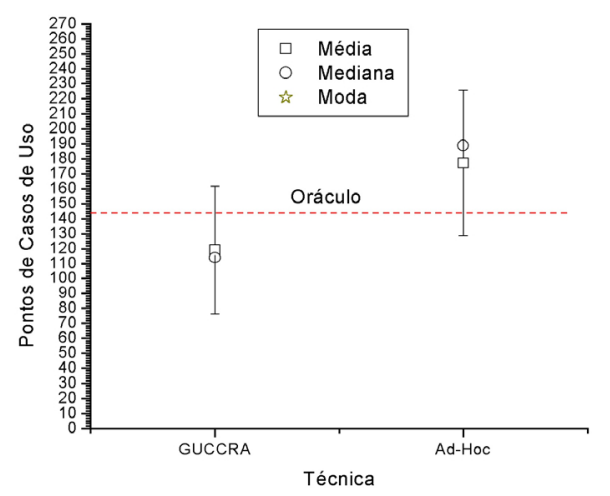

Figura 6. Documento de Requisitos C

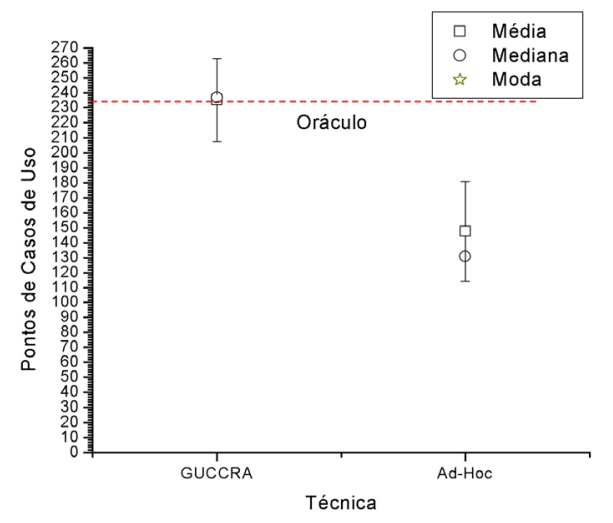

Figura 8. Documento de Requisitos E

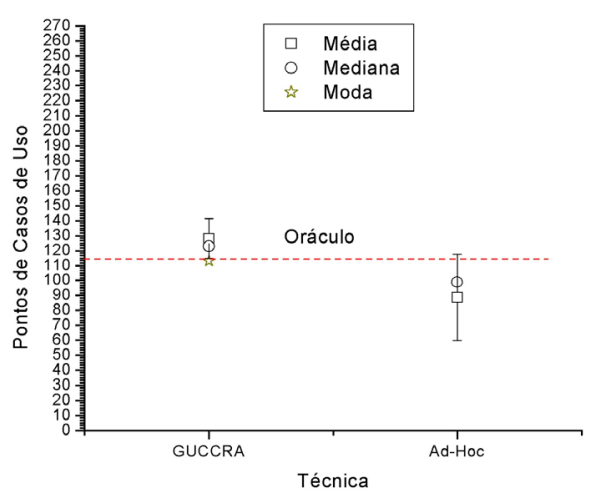

Figura 5. Documento de Requisitos B

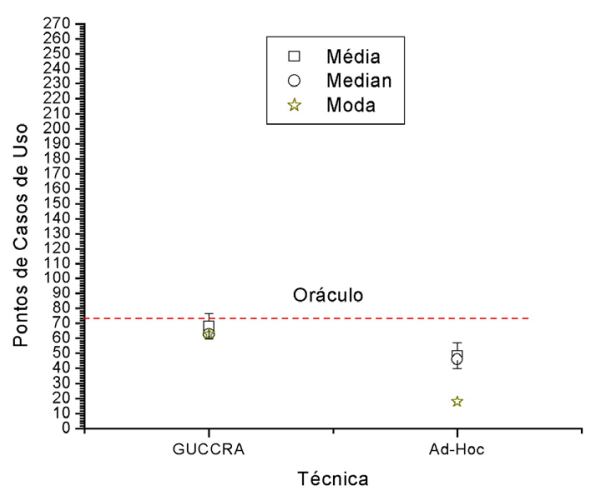

Figura 7. Documento de Requisitos D

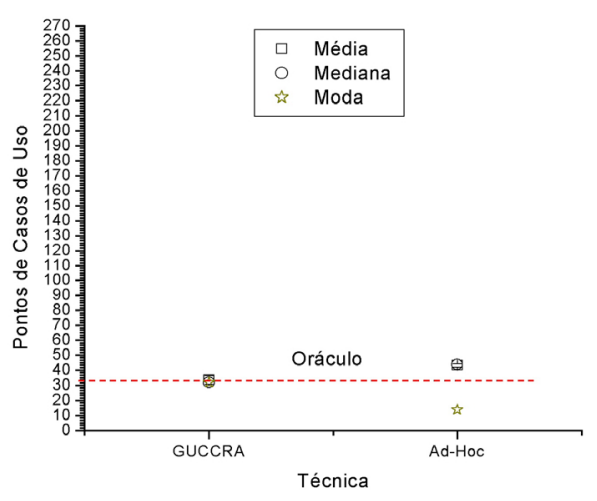

Figura 9. Documento de Requisitos F 
Algumas análises observadas nos gráficos das Figuras acima são comentadas abaixo:

- Para os documentos de requisitos B, C, E e principalmente o A, pode-se observar que o desvio padrão associado às técnicas GUCCRA $(13.2 ; 42.7 ; 27.5 ; 2.8$ respectivamente) possui uma menor variabilidade do que o desvio padrão associado à abordagem Ad-Hoc (28.9; 48.5; 33.2; 28.5 respectivamente). Esse resultado indica que há uma maior dispersão, em relação à média, dos PCU obtidos de modelos construídos com a abordagem Ad-Hoc.

- Para todos os documentos de requisitos, exceto o documento de requisitos A, os PCU dos Modelos Oráculo estão compreendidos no intervalo do desvio padrão dos PCU dos modelos construídos com as técnicas GUCCRA. Por outro lado, no caso dos modelos construídos com a abordagem Ad-Hoc, os PCU dos Modelos Oráculo não estão no intervalo do desvio padrão, para quatro dos seis documentos de requisitos utilizados (A, D, E e F). Assim, obtém-se indícios de que os PCU obtidos de modelos construídos com as técnicas GUCCRA sejam mais padronizados, permitindo estimativas próximas entre si, mesmo quando os Modelos de Casos de Uso são construídos por pessoas diferentes.

- No caso do documento de requisitos F obteve-se a ocorrência de resultados que diferem bastante dos demais: embora o intervalo de variação do desvio padrão associado com a abordagem Ad-Hoc tenha sido menor do que o associado com as técnicas GUCCRA, os PCU dos Modelos Oráculo estão compreendidos apenas no intervalo associado com as técnicas GUCCRA. Vale ressaltar que embora os PCU obtidos a partir da abordagem Ad-Hoc tenham sido parecidos, eles ficaram muito distantes dos PCU atribuídos aos Modelos Oráculo.

- Para os documentos de requisitos A, D, E e F os modelos construídos pelas técnicas GUCCRA possuem PCU bem semelhantes e próximos aos dos Modelos Oráculo. Isso pode ser observado pelo valor da moda, que foi muito próximo dos valores da média e mediana em todos os casos, o que caracteriza uma padronização dos Pontos de Casos de Uso obtidos.

\subsection{Comparação dos PCU: GUCCRA X Modelo Oráculo e Ad-Hoc X Modelo Oráculo}

O objetivo dessa análise foi avaliar se os PCU obtidos para cada modelo construído, independentemente da técnica e considerando todos os casos de uso, eram próximos dos PCU obtidos para os Modelos Oráculo.

Para essa análise foram consideradas as médias dos PCU computados para cada modelo construído tanto com as técnicas GUCCRA como com a abordagem Ad-Hoc. Avaliou-se, em porcentagem, se as médias dos PCU obtidas foram inferiores $(<)$, foram iguais $(=)$ ou superiores $(>)$ ao valor dos PCU dos Modelos Oráculo.

Os resultados da Tabela 4 mostram que, exceto para o documento B, os percentuais das médias, em relação aos PCU dos Modelos Oráculo, são muito discrepantes quando comparados os valores obtidos pelas técnicas GUCCRA e pela abordagem Ad-Hoc. Por exemplo, em relação ao documento de requisitos A, a média dos PCU do modelo criado pelas técnicas GUCCRA é inferior aos PCU do Modelo Oráculo em apenas $8 \%$ enquanto que a média dos PCU da técnica Ad-Hoc foi superior em 105\%. Essas diferenças podem prejudicar o planejamento do projeto do software e, conseqüentemente, acarretar prejuízos. 
Tabela 4. Comparação das médias dos PCU dos modelos em relação aos PCU dos Modelos Oráculo

\begin{tabular}{|c|c|c|c|c|c|c|c|c|c|}
\hline \multirow{3}{*}{\begin{tabular}{|c|} 
Documento \\
de Requisitos
\end{tabular}} & \multirow[b]{3}{*}{$\begin{array}{l}\text { PCU do } \\
\text { Oráculo }\end{array}$} & \multirow{2}{*}{\multicolumn{2}{|c|}{ Média dos PCU }} & \multicolumn{6}{|c|}{ Percentual em relação ao Modelo Oráculo } \\
\hline & & & & \multicolumn{3}{|c|}{ GUCCRA } & \multicolumn{3}{|c|}{ Ad-Hoc } \\
\hline & & GUCCRA & Ad-Hoc & $<$ & $=$ & $>$ & $<$ & $=$ & $>$ \\
\hline A & 46 & 42,67 & 94,33 & $8 \%$ & - & - & - & - & $105 \%$ \\
\hline B & 113 & 128 & 88,66 & - & - & $13 \%$ & $12 \%$ & - & - \\
\hline C & 144 & 119 & 177,33 & $18 \%$ & - & - & - & - & $23 \%$ \\
\hline D & 73 & 68 & 48,33 & $7 \%$ & - & - & $34 \%$ & - & - \\
\hline $\mathbf{E}$ & 232 & 235,33 & 147,66 & - & - & $1 \%$ & $37 \%$ & - & - \\
\hline $\mathbf{F}$ & 32 & 33,66 & 43,33 & - & - & $5 \%$ & - & - & $35 \%$ \\
\hline
\end{tabular}

Uma outra forma de mostrar os dados da Tabela 4 é através da Figura 10. Considere que o valor zero do eixo y corresponde aos PCU dos Modelos Oráculo e que as barras acima ou abaixo desse valor correspondam à porcentagem das médias dos PCU computados para os modelos construídos com as técnicas GUCCRA e com a abordagem Ad-Hoc.

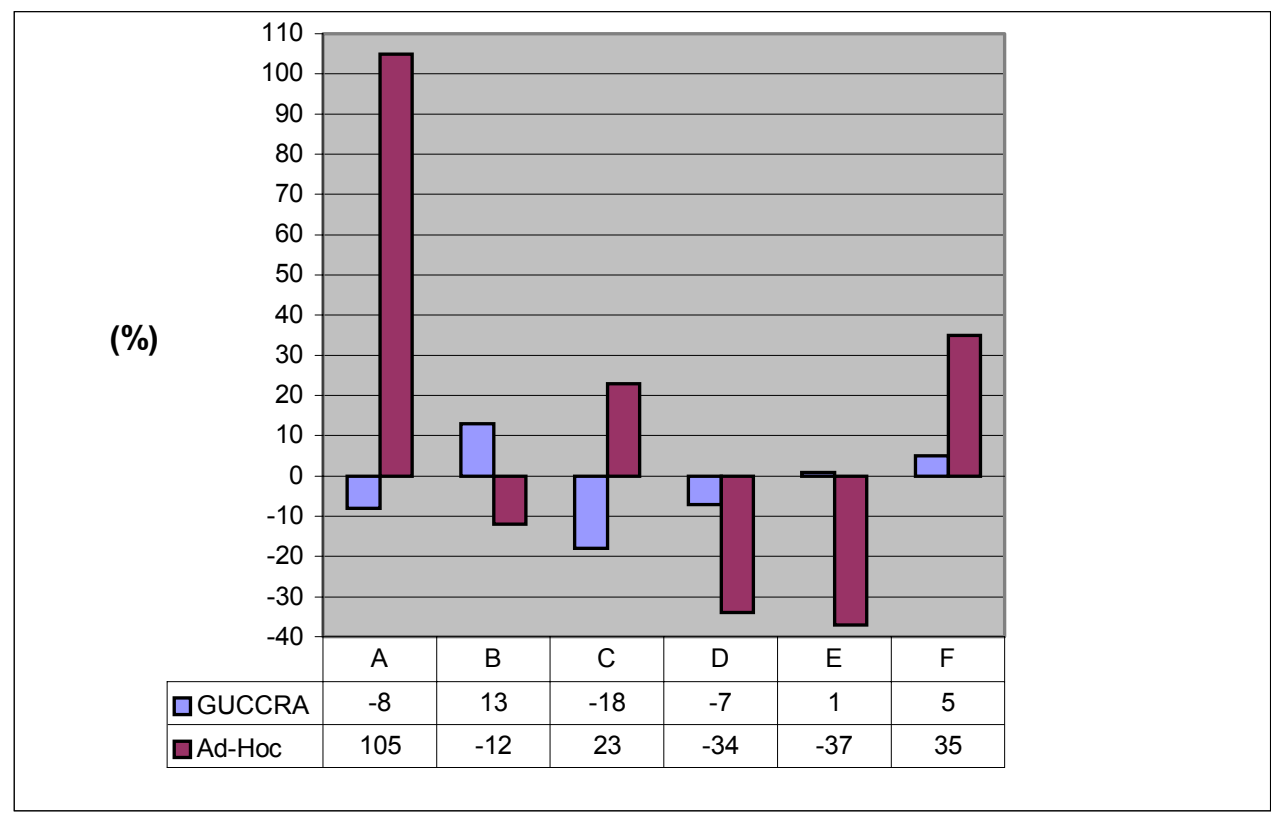

Figura 10. Porcentagem das médias dos PCU dos modelos do experimento em relação aos Modelos Oráculo, considerando todos os casos de uso identificados

Na Figura 10 pode-se observar que as diferenças das porcentagens das médias dos PCU comparadas aos PCU dos Modelos Oráculo são menores para os Modelos de Casos de Uso construídos com as técnicas GUCCRA do que para os modelos construídos com a abordagem Ad-Hoc. No caso do Documento A, por exemplo, se os PCU forem utilizados para fazer estimativas desse software, os valores advindos dos modelos gerados com as técnicas GUCCRA dariam subsídios a uma estimativa, provavelmente mais precisa, do que os PCU advindos do uso da abordagem Ad-Hoc. 


\subsection{Comparação dos PCU dos casos de uso coincidentes com os Modelos Oráculo: GUCCRA X Modelo Oráculo e Ad-Hoc X Modelo Oráculo}

O objetivo dessa análise foi avaliar, independentemente da técnica utilizada, se os PCU relativos aos casos de uso que coincidiam com casos de uso dos Modelos Oráculo eram próximos dos PCU dos Modelos Oráculo.

Para essa análise foram consideradas as médias dos PCU relativas aos casos de uso coincidentes com casos de uso dos Modelos Oráculo. Avaliou-se, em porcentagem, se as médias dos PCU obtidas foram inferiores $(<)$, foram iguais $(=)$ ou superiores $(>)$ aos valores dos PCU dos Modelos Oráculo. Na Tabela 5 são mostrados os resultados obtidos.

Tabela 5. Comparação das médias dos PCU dos casos de uso coincidentes em relação aos PCU dos Modelos Oráculo

\begin{tabular}{|c|c|c|c|c|c|c|c|c|c|}
\hline \multirow{3}{*}{\begin{tabular}{|c|} 
Documento \\
de Requisitos
\end{tabular}} & \multirow[b]{3}{*}{$\begin{array}{l}\text { PCU do } \\
\text { Oráculo }\end{array}$} & & & \multicolumn{6}{|c|}{ Percentual em relação ao Modelo Oráculo } \\
\hline & & \multicolumn{2}{|c|}{ Média dos PCU } & \multicolumn{3}{|c|}{ GUCCRA } & \multicolumn{3}{|c|}{ Ad-Hoc } \\
\hline & & GUCCRA & Ad-Hoc & $<$ & $=$ & $>$ & $<$ & $=$ & $>$ \\
\hline $\mathbf{A}$ & 46 & 41 & 21,66 & $11 \%$ & - & - & $53 \%$ & - & - \\
\hline B & 113 & 119,66 & 41,33 & - & - & $5 \%$ & $64 \%$ & - & - \\
\hline $\mathbf{C}$ & 144 & 115,66 & 85,66 & $20 \%$ & - & - & $51 \%$ & - & - \\
\hline D & 73 & 66,33 & 29,66 & $10 \%$ & - & - & $60 \%$ & - & - \\
\hline $\mathbf{E}$ & 232 & 218,66 & 116 & $6 \%$ & - & - & $50 \%$ & - & - \\
\hline $\mathbf{F}$ & 32 & 32 & 16,66 & - & $100 \%$ & - & $48 \%$ & - & - \\
\hline
\end{tabular}

Os resultados da Tabela 5 mostram que, para o documento de requisitos $\mathrm{F}$, o valor médio dos PCU obtidos nos modelos construídos com as técnicas GUCCRA correspondeu exatamente ao valor dos PCU do Modelo Oráculo (32). Com relação à abordagem Ad-Hoc, esse valor foi inferior em $48 \%$. Com base no experimento relatado na Seção 3 pode-se afirmar que a coincidência na média obtida em relação às técnicas GUCCRA não se restringe apenas a uma coincidência numérica relativa aos PCU. Essa coincidência se deu efetivamente nos modelos construídos, ou seja, com o uso das técnicas GUCCRA os participantes construíram modelos muito semelhantes aos Modelos Oráculo.

Uma forma gráfica de representação dos dados da Tabela 5 pode ser visualizada no gráfico da Figura 11. Considere que o valor zero do eixo y corresponde aos PCU dos Modelos Oráculos e que as barras acima ou abaixo desse valor correspondam à porcentagem das médias dos PCU computados para os modelos construídos com as técnicas GUCCRA e com a abordagem Ad-Hoc.

Na Figura 11 pode-se observar que, para todos os documentos de requisitos, as médias dos PCU foram significativamente inferiores em relação aos PCU dos Modelos Oráculo quando do uso da abordagem Ad-Hoc. Já para as técnicas GUCCRA, desconsiderando o documento F em que os PCU foram iguais, para os demais documentos de requisitos os valores inferiores ou superiores foram muito próximos dos valores dos Modelos Oráculo. Isso mostra que os modelos construídos com essas técnicas são muito mais padronizados, independentemente de quem os construa. 


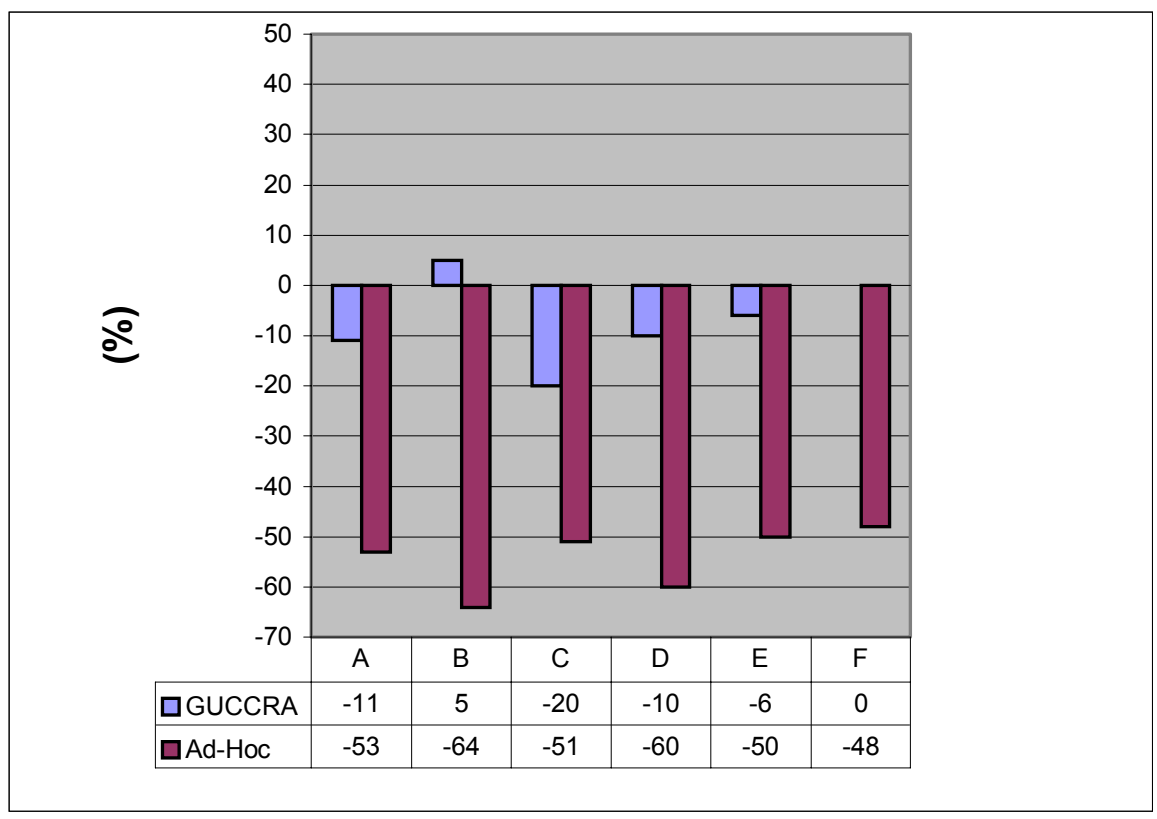

Figura 11. Porcentagem das médias dos PCU dos modelos do experimento em relação aos Modelos Oráculo, considerando os casos de uso coincidentes com os casos de uso dos Modelos Oráculo

\section{Conclusões}

Apresentou-se neste artigo um estudo sobre a utilização da métrica Pontos de Casos de Uso em modelos gerados com a aplicação das técnicas de leitura GUCCRA e da abordagem AdHoc. Esses modelos foram construídos a partir de seis documentos de requisitos, os quais foram elaborados com base no Padrão IEEE.

Os resultados obtidos mostraram que:

- Os Pontos de Casos Uso dos modelos construídos com as técnicas GUCCRA, para o mesmo documento de requisitos, são mais aproximados, isto é, a variabilidade dos Pontos de Casos de Uso é menor quando comparada aos Pontos de Casos Uso dos modelos construídos com a abordagem Ad-Hoc.

- Os Pontos de Casos Uso dos modelos construídos com as técnicas GUCCRA assemelham-se aos Pontos de Casos Uso de um modelo oráculo que foi construído para cada um dos documentos de requisitos.

Portanto, mostrou-se que a contagem dos Pontos de Casos de Uso pode ser diretamente influenciada e a acurácia desse valor pode ser efetivamente melhorada com uma maior sistematização na construção de modelos de casos de uso, comprovando a necessidade dessa sistemática como foi observado no trabalho de Andrade [8].

Dessa forma, melhorando os valores dos Pontos de Casos de Uso, é muito provável que as estimativas geradas para o sistema produzam dados mais próximos da realidade, melhorando também a qualidade da atividade de Planejamento.

Para a apresentação desse estudo, o artigo também mostrou, resumidamente, as etapas das técnicas GUCCRA e os resultados de um experimento que foi conduzido anteriormente, para fazer uma avaliação da utilização dessas técnicas e da abordagem Ad-Hoc na construção de Modelos de Casos Uso. Tais resultados evidenciaram que a sistematização do processo de construção dos Modelos de Casos de Uso leva à geração de modelos mais padronizados (no 
sentido de que, pessoas diferentes elaboram modelos semelhantes para o mesmo documento de requisitos) e menos dependentes da subjetividade e experiência das pessoas.

\section{Referências}

[1] Paulk,Mark; Curtis, B.; Chrissis, M.B. et al. "Capability Maturity Model for Software, Version 1.1”, SEI Techinal Report CMU/SEI-93-TR-24, 1993.

[2] CMMI,2002 - "Capability Maturity Model Integration Version 1.1", (CMMISE/SW/IPPD/SS, V1.1), SEI Techinical Report CMU/SEI-2002-TR-011.

[3] ISO/IEC TR 15504: 1998 - Information Technology - Software Process Assessment parts 1-9, International Standard Organization, 1998.

[4] Garmus, David and Heron, David. Function Point Analysis, Addison-Wesley, 2000.

[5] Boehm, B. Software Engineering Economics, Prentice-Hall, 1981.

[6] Boehm, B. "Anchoring the Software Process", IEEE Software, vol. 13, no. 4, pp. 7382, July 1996.

[7] Schneider, G. and Winters, J. P. Applying Use Cases, A Practical Guide. Second Edition, Addison-Wesley, 2001.

[8] Andrade, E. L. P. Pontos de Casos de Uso e Pontos de Função na gestão de estimativa de tamanho de projetos de software orientado a objetos.Dissertação de Mestrado. Universidade Católica de Brasília, 2004.

[9] Belgamo, A. GUCCRA: Técnicas de Leitura para Construção de Modelos de Casos de Uso e Análise do Documento de Requisitos. Dissertação de Mestrado. Departamento de Computação, Universidade Federal de São Carlos. Abril, 2004.

[10] Cockburn, A. Writing Effective Use Cases. Boston MA: Addison-Wesley, 2001.

[11] Object Managmente Group. Unified Modeling Language Specification. Version 1.5. 2003. URL: http://www.omg.org/uml/.

[12] Kulak, D.; Guiney, E. Use Cases: Requirements in Context. Addison-Wesley, 2000.

[13] Ryser, J., Glinz, M. "Using Dependency Charts to Improve Scenario-Based Testng", 17th International Conference on Testing Computer Software - TCS'2000, Washington, D.C., 2000.

[14] Anchor, Ben. C., Rolland, C., Maiden, N. A. M. and Souveyet, C. "Guiding Use Case Authoring: Results of an Empirical Study", Proc. of the $4^{\text {th }}$ International Symposium on Requirements Engineering, 1999.

[15] IEEE Recommended Practice for Software Requirements Specifications, Std 8301998, 1998.

[16] Ribu, Kirsten. Estimating Object-Oriented Software Projects with Use Cases. Master Thesis. Departament of Informatic, University of Oslo. Norway. November, 2001.

[17] Anda, Bente. Dreiem, Hege. Sjoberg, Dag I.K. and Jorgensen, Magne. "Estimating Software Development Effort based on Use Cases - Experiences from Industry", Proc. Of the $4^{\text {th }}$ Internation Conference on The Unified Modeling Language. Toronto, Canadá, October, 2001.

[18] Anda, Bente. "Comparing Effort Estimates Based on Use Case Points with Expert Estimates”, Empirical Assessesment in Software Engineering, Keele, UK, April, 2002. 